1 Resistance of Anopheles gambiae to the new insecticide clothianidin

2 associated with unrestricted use of agricultural neonicotinoids in Yaoundé,

3 Cameroon

4

5 Caroline Fouet ${ }^{1}$, Ayukarah F. Ashu ${ }^{1,2}$, Marilene M. Ambadiang1,2, Williams Tchapga1,

6 Charles S. Wondji ${ }^{1,3,4}$ and Colince Kamdem ${ }^{1 *}$

8 1-Centre for Research in Infectious Diseases (CRID), P.O. Box 13591, Yaoundé,

9 Cameroon

10 2-Department of Biochemistry, Faculty of Science, University of Yaoundé 1, P.O. Box

11 812, Yaoundé, Cameroon

12 3-Vector Biology Department, Liverpool School of Tropical Medicine (LSTM),

13 Pembroke Place, Liverpool L3 5QA, UK

14 4LSTM Research Unit at CRID, P.O. Box 13591, Yaoundé, Cameroon

15

16

17 *Correspondence: colincek@crid-cam.net,kamdem_d@yahoo.com

18

19 
20 Abstract

21 Background: New active ingredients, mostly repurposed from the agricultural

22 sector, are gradually being introduced into malaria vector control programs. Prior to

23 scaling up novel insecticides, it is vital to assess if their use in agriculture has

24 already selected mosquito populations with reduced susceptibility in some areas.

25 The aim of the present study was to determine if the use of neonicotinoids in

26 agricultural pest management could drive resistance to clothianidin-a

27 neonicotinoid recently prequalified by WHO for indoor residual spraying.

Methods: We carried out intensive sampling of Anopheles gambiae, An. coluzzii and Culex sp. larvae from a large suburban farm and from 4 surrounding locations

31 belonging to the rural and urban ecosystems of Yaoundé, Cameroon. Using WHO

32 tube tests and CDC bottle bioassays, we tested the recommended diagnostic doses of

33 clothianidin against field-collected populations using two laboratory strains as

34 susceptible controls. We exposed adult mosquitoes to the insecticide for $1 \mathrm{~h}$ and

35 monitored mortality rates for seven consecutive days.

37 Results: An. coluzzii and Culex sp. mosquitoes were fully susceptible to clothianidin

38 reaching $100 \%$ mortality by day 3. By contrast, An. gambiae populations revealed

39 heterogeneous profiles ranging from susceptibility to strong resistance. Populations

40 from the suburban farm, where neonicotinoids are routinely used, displayed the

41 lowest mortality rate $(46.5 \% \pm 5.65)$ and were capable of surviving $5 \mathrm{X}$ and $10 \mathrm{X}$ the

42 diagnostic dose of clothianidin. When clothianidin was tested in combination with

43 the synergist piperonyl butoxide, mortality of resistant populations increased from

$44 \quad 46.5 \%$ to $92.7 \%$ implying that Cytochrome P450 enzymes play an integral role in

45 the genetic mechanism underlying clothianidin resistance in An. gambiae.

47 Conclusions: These findings show that small clusters of resistance to clothianidin

48 may exist around farmlands where agricultural neonicotinoids are being used.

49 Patches of intensive agriculture should be particularly monitored in indoor residual

50 spraying target areas. 
52 Key words: Anopheles gambiae, clothianidin, resistance, cytochrome P450s, indoor

53 residual spraying, neonicotinoids.

Introduction

56 Research is ongoing to diversify malaria control techniques by developing new tools

57 that could reduce the reliance of intervention measures on chemical insecticides

$58[1,2]$. However, insecticidal interventions remain the mainstay of current prevention

59 and control strategies. Notably, the scale-up of Indoor Residual Spraying (IRS) and

60 Long-Lasting Insecticidal Nets (LLINs) have dramatically reduced malaria incidence

61 and mortality in Sub-Saharan Africa over the last two decades [3]. Unfortunately,

62 this progress might become difficult to preserve in the future if the rapid spread of

63 insecticide resistance that has been observed among malaria vectors is not

64 effectively managed $[4,5]$. For this reason, WHO continues to underscore the critical

65 need for all countries with ongoing malaria transmission to develop and apply

66 effective insecticide resistance management strategies [6,7].

68 Only a handful of insecticides are prequalified by WHO for core interventions for 69 malaria vector control [8]. In the face of widespread insecticide resistance, control

70 programs have had little choice but to switch between active ingredients in order to

71 preserve the efficacy of intervention measures. This sequential deployment of

72 insecticides is often associated with substantial increases in intervention costs as

73 well as the development of multiple forms of resistance that are more difficult to

74 manage [9]. For example, countries have progressively adopted more expensive

75 alternatives such as carbamates and organophosphates for IRS to mitigate mosquito

76 resistance to pyrethroids. This switch has been correlated with a decline in IRS

77 coverage from a peak of 5\% in 2010 to $2 \%$ in 2018 [10]. To address this issue, the

78 WHO's global plan for insecticide resistance management in malaria vectors has

79 recommended the deployment of active ingredients in rotations, combinations,

80 mosaics and mixtures [6,9]. To enhance the potential effectiveness of this

81 management strategy, a short list of new active ingredients with distinct modes of 
82 action has been evaluated and tested for the control of mosquito vector populations

83 that are already resistant to chemicals routinely used for IRS and LLLINs [8].

85 Clothianidin is a neonicotinoid manufactured into two new IRS formulations that 86 have been evaluated and prequalified by the WHO [8]. Those are SumiShield 50WG ${ }^{\circledR}$

87 (50\% clothianidin) developed by Sumitomo Chemical and Fludora ${ }^{\circledR}$ Fusion (Bayer 88 Environmental Science) which is a mixture of $500 \mathrm{~g} / \mathrm{kg}$ clothianidin and $62.5 \mathrm{~g} / \mathrm{kg}$ 89 deltamethrin $[11,12]$. Neonicotinoid pesticides were discovered in the late 1980s 90 and have since become the most widely used class of insecticides worldwide, with 91 large-scale applications ranging from plant protection, veterinary application, and 92 biocides to invertebrate pest control in fish farming [13]. Currently, 8 registered 93 neonicotinoid insecticides are available commercially worldwide [13]. These 94 insecticides target the nicotinic acetylcholine receptor (nAChR) in the insect central 95 nervous system and cause over-stimulation, which may result in paralysis and 96 death. This mode of action is distinct from that of the other neurotoxic insecticides 97 such as pyrethroids routinely used for IRS and LLLINs over the last decades. For this 98 reason, clothianidin formulations are potentially suitable for inclusion in malaria 99 vector control, particularly in areas with high pre-existing resistance to multiple 100 insecticide classes. Importantly, field evaluations of SumiShield 50WG and Fludora

101 Fusion have reported promising results, both formulations showing effective 102 insecticidal action against different vector species as well as durable residual 103 activity on diverse surfaces [14-17].

105 In preparation for rollout of clothianidin formulations as part of national IRS 106 rotation strategies, the U.S. President's Malaria Initiative has conducted large-scale 107 susceptibility testing of wild anopheline populations from 16 African countries [18]. 108 This investigation revealed that populations of the most important vectors of 109 Plasmodium parasites across the continent-An. gambiae, An. coluzzii, An. arabiensis 110 and An. funestus - were susceptible to filter papers impregnated with SumiShield $11150 \mathrm{WG}$ at a diagnostic dose of $2 \% \mathrm{w} / \mathrm{v}$ clothianidin. While the findings of this study 112 are encouraging, continental-scale surveys should be complemented with targeted 
113 testing of populations from agricultural regions where Anopheles mosquitoes are

114 more likely to develop resistance to neonicotinoids. The association between

115 agricultural usage of pesticides and development of insecticide resistance in

116 Anopheles mosquitoes is widely documented (e.g., [19-22] reviewed in [23]). It has

117 been hypothesized that pesticide residues contaminate aquatic habitats and

118 contribute to the selection of resistance genes among non-target insect populations

119 including mosquito larvae. Neonicotinoids are highly soluble in water and can

120 persist for months in aerobic soils, therefore making contamination of mosquito

121 breeding sites following agricultural use highly likely [24]. Published information on

122 the usage of neonicotinoids across Sub-Saharan Africa is lacking, but preliminary

123 reports from Cameroon, Tanzania and Ivory Coast suggest that hundreds of

124 commercial formulations of neonicotinoids have been registered for use in crop

125 protection [25-27]. Consequently, prior to field use of clothianidin, it is imperative

126 to evaluate if the widespread application of neonicotinoids in agriculture is

127 contributing to the emergence of resistant vector populations in some areas. Here

128 we aimed to address the likelihood of clothianidin resistance driven by agricultural

129 neonicotinoids in Anopheles and Culex mosquito populations. To do so, we assessed

130 susceptibility in wild populations from five locations centered on one of the largest

131 urban farms in Cameroon's political capital, Yaoundé, using laboratory bioassays.

132 Our results reveal strong clothianidin resistance in An. gambiae populations that

133 thrive in standing water within farm plots where several neonicotinoid insecticides

134 are routinely used. This finding suggests that mosquito susceptibility and

135 neonicotinoid usage should be particularly monitored around agricultural patches

136 in IRS target areas.

138 Methods

\section{Study sites}

141 To investigate the effect of agricultural neonicotinoids on clothianidin susceptibility

142 in mosquitoes, we tested populations from a large farm located in Nkolondom $143 \quad\left(3^{\circ} 56^{\prime} 43^{\prime \prime} \mathrm{N}, 11^{\circ} 31^{\prime} 01^{\prime \prime} \mathrm{E}\right)$, a suburban neighborhood of Yaoundé, the capital city of 
144 Cameroon (Figure 1A). For comparison, we also tested mosquito populations from

145 two neighborhoods situated at the center of Yaoundé and from two rural sites at the

146 outskirts of the city [28] (Fig. 1A). Nkolondom is located in the northern part of

147 Yaoundé-approximately $7 \mathrm{~km}$ from the center of the city. Since 1985, artisanal

148 agriculture has gained considerable momentum in a swampy area of this locality,

149 which currently attracts at least one hundred farmers [29]. At present, this

150 suburban farm encompasses 100-200 mosaics of $\sim 20 \mathrm{~m}^{2}$ adjacent plots and has

151 become the main production site of celery, amaranth, lettuce and other aromatic

152 herbs in Yaoundé (Fig. 1B). Farming activities are conducted year-round, and crops

153 are intensively watered either manually or using artisanal irrigation schemes.

154 Consequently, standing water accumulates in the fields where very high densities of

155 An. gambiae and Culex sp larvae can be collected throughout the year.

\section{Mosquito collection and rearing}

158 Mosquito collections took place during the long rainy season and part of the long

159 dry season: from July 2019 to January 2020 and from May 2020 to July 2020. We

160 sampled mosquito larvae across the study sites using the standard dipping method

161 [30]. Larvae were collected from standing water within the farms in Nkolondom,

162 from relatively shallow ruts in rural areas and from polluted breeding sites at the

163 center of Yaoundé city. Larvae were transported in closed containers to the CRID's

164 insectary and reared to adults under standard laboratory conditions. An. gambiae

165 and An. coluzzii are the dominant malaria vector species in Yaoundé where their

166 respective habitats have been well characterized in past surveys [28,31]. An. coluzzii

167 is found exclusively in the most urbanized neighborhoods while An. gambiae is the

168 only species present in the rural areas. Sampling was conducted to collect and test

169 one species per location. We collected and tested Culex populations that occur in the

170 same breeding sites as An. gambiae in Nkolondom to compare their susceptibility

171 profiles. For bioassay tests, we used two laboratory strains as susceptible controls:

172 An. gambiae Kisumu and An. coluzzii Ngousso. Both strains are known to be

173 susceptible to pyrethroid, carbamate, organochlorine and organophosphate

174 insecticides. We maintained all life-cycle stages of laboratory strains and wild 
175 mosquito populations under standard insectary conditions $\left(25 \pm 2{ }^{\circ} \mathrm{C}, 80 \%\right.$ relative

176 humidity, light:dark cycles of 12:00 h each) and we provided adults with 10\% sugar

177 solution.

179 CDC bottle tests

180 We tested the susceptibility of adult mosquitoes against a diagnostic dose of 181 clothianidin using CDC bottle assays according to published guidelines [34]. A recent

182 study showed that $150 \mu \mathrm{g} / \mathrm{ml}$ clothianidin was a suitable diagnostic dose-the

183 lowest concentration of active ingredient that kills $100 \%$ of individuals of a

184 susceptible laboratory strain within $24 \mathrm{~h}$ of insecticide exposure [35]. To assess the

185 intensity of resistance, we also determined the mortality rates of wild mosquitoes

186 exposed to $5 \mathrm{X}$ and $10 \mathrm{X}$ the diagnostic dose. We prepared stock solutions of

187 clothianidin at $1 \mathrm{X}, 5 \mathrm{X}$, and $10 \mathrm{X}$ the diagnostic dose using technical grade

188 clothianidin (PESTANAL ${ }^{\circledR}$, analytical standard, Sigma-Aldrich, Dorset, United

189 Kingdom) and absolute ethanol as solvent. The solutions were conserved at $4^{\circ} \mathrm{C}$ in

190 the dark for $24 \mathrm{~h}$ before use to maximize the solubility of clothianidin. Each

191 Wheaton 250-ml bottle and its cap were coated with $1 \mathrm{ml}$ of clothianidin solution

192 following the CDC protocol. For each bioassay, we used a maximum of four test

193 bottles coated with clothianidin and two control bottles coated with $1 \mathrm{ml}$ of absolute

194 ethanol. Occasionally, depending on the availability of mosquitoes, we used less than

195 four test bottles and only one control. All bottles were wrapped in aluminum foil

196 and allowed to dry for $24 \mathrm{~h}$ for complete evaporation of solvent before use. Bottles

197 were not reused but were washed three times in warm soapy water and allowed to

198 dry for $24 \mathrm{~h}$ before being coated.

199 We carried out susceptibility tests as follows: 20 to 25 3-5-day-old females were

200 aspired from mosquito cages and gently released into one of the test bottles where

201 they were exposed to the active ingredient or into control bottles containing ethanol

202 for 60 minutes. Following exposure, mosquitoes were transferred into a paper cup

203 and provided with 10\% sugar solution. Knockdowns were scored and mortality was

204 monitored every day for seven consecutive days under standard laboratory 
205 conditions. When a population displayed signals consistent with resistance, we 206 carried out repeat tests for confirmation.

\section{WHO tube tests}

209 We used WHO tube tests to assess the susceptibility of populations showing the 210 lowest mortality rate in CDC bottle bioassays to SumiShield 50WG, which contains $21150 \%(\mathrm{w} / \mathrm{w})$ clothianidin [36]. We carried out repeat tests of these resistant

212 populations using a susceptible field population and a susceptible lab strain as 213 negative controls. We used a diagnostic dose of 2\% w/v clothianidin (13.2 mg active 214 ingredient per paper) based on guidance from Sumitomo Corporation and the US 215 President's Malaria Initiative. This dose has been successfully used to test the 216 toxicity of clothianidin towards wild anopheline populations from Sub-Saharan 217 Africa [18,37]. We prepared a stock solution by diluting $264 \mathrm{mg}$ SumiShield 50WG in $21820 \mathrm{ml}$ distilled water. We impregnated Whatman filter papers $(12 \mathrm{x} 15 \mathrm{~cm})$ containing $21913.2 \mathrm{mg}$ clothianidin each using $2 \mathrm{ml}$ of the insecticide solution as described in [38]. 220 We prepared control filter papers using $2 \mathrm{ml}$ of distilled water. Treated filter papers 221 were allowed to dry overnight and were kept in foil at $4^{\circ} \mathrm{C}$ until use. To carry out 222 bioassay tests, we aspired 20 to 25 3-5-day-old female mosquitoes from mosquito 223 cages that we introduced into each of the test tubes containing clothianidin224 impregnated papers. We concomitantly released 20-25 mosquitoes into each 225 control tube containing a water-impregnated paper. After 60 minutes of exposure, 226 mosquitoes were transferred to holding tubes and provided with 10\% sugar 227 solution, and knockdown time was recorded. As with CDC bottle tests mortality was 228 scored every day until day 7.

\section{Synergist bioassays}

231 To investigate the potential role of oxidases in the genetic mechanisms underlying

232 the reduced susceptibility to clothianidin, we used CDC bottle assays to determine 233 the efficacy of the diagnostic dose in combination with the synergist PBO (4\%). PBO 234 is an inhibitor of oxidases and non-specific esterases and enhances cuticular 235 penetration of the insecticide into the insect [39]. A solution of $400 \mu \mathrm{g} / \mathrm{ml} \mathrm{PBO}$ was 
236 prepared by diluting PBO with absolute ethanol. The solution was mixed and stored 237 in the dark at $4^{\circ} \mathrm{C}$ before use. Wheatman bottles and their cap were coated with $1 \mathrm{ml}$

238 of the PBO solution, wrapped in aluminum foil and allowed to dry for $24 \mathrm{~h}$ before the 239 test. 100 to 150 female mosquitoes aged between 3 and 5 days were pre-exposed 240 for $60 \mathrm{~min}$ to PBO-coated bottles or to control bottles coated with ethanol.

241 Mosquitoes were then gently removed from the bottles and introduced in batches of

24220 to 25 mosquitoes into new bottles coated with $1 \mathrm{X}$ clothianidin solution for the

243 tests or with ethanol for the controls. After 60 minutes, mosquitoes were

244 transferred into a paper cup and provided with 10\% sugar solution. Knockdowns

245 were scored and mortality was monitored every day for seven consecutive days in

246 standard laboratory conditions. We compared mortality rates with or without the

247 synergist to determine to what extent inhibiting oxydases affected the level of 248 susceptibility.

250 Data analysis

251 We used mortality rates to evaluate the efficacy of clothianidin against laboratory 252 and field mosquitoes. All tests with mortality $>20 \%$ at day 7 in controls were 253 discarded. We used Abbott's formula to correct mortality rates of tests when 5\%$25420 \%$ individuals died between day 1 and day 7 in the corresponding controls. 255 Following the WHO guidelines on insecticide susceptibility, mosquito populations 256 were considered susceptible if mortality at day 7 was $\geq 98 \%$ and resistant if 257 mortality was less than 90\%. Mortality rates between $90 \%$ and $97 \%$ indicate that 258 the presence of resistant genes in the vector population must be confirmed by 259 additional tests [36]. We used Wilcoxon test to determine if mean mortality rates 260 were significantly different among tested populations. We performed all analyses 261 using the R software [40].

\section{Results}


267 Across the 5 study sites, we used CDC bottle bioassays to test the diagnostic dose of 268 clothianidin against a total of 1665 wild mosquitoes belonging to three species: $A n$.

269 gambiae $(\mathrm{n}=817)$, An. coluzzii $(\mathrm{n}=673)$ and Culex sp $(\mathrm{n}=132)$. To validate the CDC 270 bottle bioassay protocol [35], we first analyzed susceptibility in 554 individuals of 271 two laboratory colonies: An. gambiae Kisumu (n = 326) and An. coluzzii Ngousso (n 272 =228). Results showed that the two laboratory strains were fully susceptible to 273 clothianidin, reaching 100\% mortality between the first and the third day post 274 exposure (Fig. 2A). Among field mosquitoes, Culex sp. populations were the most 275 susceptible to clothianidin, achieving 100\% mortality in approximately $24 \mathrm{~h}$ (Fig. 276 2A). An. coluzzii populations from urban areas were also susceptible to the active 277 dose of clothianidin, although 100\% mortality was reached only between the fourth 278 and the seventh day. In An. gambiae by contrast, the overall mortality was only 58.2 $279 \pm 5.2 \%$ in all 817 individuals tested from 4 locations, suggesting that populations of 280 this species have developed resistance to clothianidin. As previously reported in a 281 continental survey of clothianidin susceptibility in Anopheles throughout 16 African 282 countries [18], knockdown at 60 min was generally low, except field populations of 283 An. coluzzii (81.8 $\pm 2.9 \%)$, Mortality within 60 min of exposure ranged from $42 \pm$ $28411.0 \%$ to $53.9 \pm 9.9 \%$ for the lab strains and from $32.1 \pm 5.0 \%$ to $36.5 \pm 12.2 \%$ 285 among field populations (Fig. 3A). Consistent with their reduced susceptibility to 286 clothianidin, wild populations of An. gambiae displayed the lowest knockdown (32.1 $287 \pm 5.0 \%$ ). Across our sampling sites, An. gambiae populations occur mainly in rural 288 and suburban areas and are absent in the most urbanized regions [28,31]. Our 289 findings suggest that farming activities associated with the usage of agricultural 290 neonicotinoids, which are common in rural and suburban areas of Yaoundé, may be 291 contributing to the selection of clothianidin resistance in An. gambiae mosquitoes

\section{2- Association between usage of agricultural neonicotinoids and clothianidin}

294 resistance in An. gambiae

296 To examine the link between usage of agricultural neonicotinoids and the 297 development of clothianidin resistance in An. gambiae, we compared susceptibility 
298 profiles of populations from the suburban farm and those from the rural sites. 299 Mortality rates varied along a gradient, ranging from full susceptibility in some 300 populations from rural areas to strong resistance in samples from the Nkolondom

301 farm where only $46.5 \pm 5.7 \%$ of exposed individuals died between the first and the 302 seventh day of holding period (Fig. 2B). There was no statistically significant 303 difference in mortality among sites except between the suburban farm and each of 304 the rural sites (Wilcoxon tests, $\mathrm{p}<0.02$ ). Knockdown at $60 \mathrm{~min}$ in Nkolondom 305 samples was also lowest (20.1 $\pm 4.1 \%)$ and significantly different from values 306 obtained with rural populations (Wilcoxon tests, p < 0.03) (Fig. 3B). Although these 307 observations still need to be replicated and validated across other agricultural 308 settings, our data suggest that resistance to agricultural neonicotinoids is the likely 309 driver of reduced clothianidin susceptibility observed in An. gambiae populations 310 from the Nkolondom farms. During our field survey in Nkolondom, we collected 311 used cans of imidacloprid and acetamiprid (Fig. 1C). We also witnessed the 312 intensive application of those products in mixtures of pesticides, which 313 corroborates observations indicating significant usage of neonicotinoids in some 314 agricultural hotspots in Cameroon (Fig. 1D) [25]. Nevertheless, we detected signals 315 consistent with resistance in a rural site without obvious evidence of agricultural 316 activities involving the use of neonicotinoids-Zamengoué, 356'29" N, $11^{\circ} 27^{\prime} 07^{\prime \prime}$ 317 E-, mortality: $72 \pm 12.0 \%$ at day 7 . This finding might be due to dispersal of 318 populations from Nkolondom located about $3 \mathrm{~km}$ from Zamengoué or from another 319 unidentified agricultural site. This result could also suggest that other 320 environmental factors in addition to agricultural activities may contribute to the 321 selection of clothianidin-resistant vector populations. Also, we noted that Culex sp 322 samples collected from the breeding sites where resistant An. gambiae larvae thrive 323 in Nkolondom were susceptible to clothianidin. This observation lends support to 324 the view that cross-resistance to agricultural neonicotinoids is species-specific and 325 might be only one among a list of mechanisms underlying the adaptation of insect 326 populations to this class of insecticide in agricultural regions. 
328 3- Clothianidin resistance is strong in An. gambiae populations from Nkolondom

331 To assess the strength of resistance driven by agricultural neonicotinoids, we used 332 CDC bottle assays to evaluate the mortality rates of resistant populations from 333 Nkolondom against 5X to 10X the diagnostic dose of clothianidin. Results indicated 334 that only $37.5 \pm 7.7 \%$ and $77.5 \pm 12.6 \%$ of populations exposed to $5 \mathrm{X}$ and $10 \mathrm{X}$ the 335 diagnostic dose of clothianidin, respectively, died between the first and the seventh 336 day following exposure (Fig. 3C). According to WHO guidelines, when resistance is 337 confirmed at the 5X and especially at the 10X concentrations, operational failure is 338 likely [36]. To determine to what extent strong clothianidin resistance could affect

339 the efficacy of manufactured formulations, we used WHO tube tests to measure the 340 susceptibility of farm mosquitoes to SumiShield 50 WG. This formulation

341 corresponds to $\sim 24 \mathrm{X}$ the diagnostic dose of clothianidin used in CDC bottle assays.

342 We used the susceptible Ngousso strain as negative controls. As expected, the lab 343 strain and the control field populations were susceptible to SumiShield 50 WG, 344 reaching $100 \%$ after 48 h (Fig. 2C). By contrast, mortality rates of An. gambiae 345 populations from the Nkolondom farm plateaued at $75.4 \pm 3.5 \%$ after 7 days of 346 holding period. This finding suggests that resistant populations from Nkolondom

347 are currently capable of surviving very high concentrations of clothianidin used in 348 SumiShield $50 \mathrm{WG}$ and could be challenging to control with the new IRS 349 formulations.

351 4- Oxydases play a central role in clothianidin resistance in An. gambiae

353 To start investigating the genetic mechanisms underlying the resistance to 354 clothianidin in An. gambiae, especially the role of cytochrome P 450 enzymes, we 355 carried out CDC bottle assays to evaluate the toxicity of the diagnostic dose of 356 clothianidin in combination with PBO. We found that mortality among clothianidin357 resistant populations from the agricultural site increased from $46.5 \pm 5.7 \%$ without 358 PBO to $92.7 \pm 3.7 \%$ in presence of the synergist (Wilcoxon test: $p=0.015$ ) (Fig. 2D). 
359 Thus, when clothianidin is combined with PBO, resistant populations almost fully

360 recovered their susceptibility, which indicates that cytochrome P450 enzymes play

361 a major role in the development of resistance in An. gambiae.

\section{Discussion}

365 The present study aimed to determine if the intensive use of neonicotinoid

366 insecticides in some agricultural regions in Sub-Saharan Africa could undermine the

367 efficacy of new IRS formulations containing clothianidin. Indeed, the scale up of

368 insecticides in large-scale vector control measures combined with rapid

369 urbanization and extensive agriculture have exposed Anopheles mosquitoes to a

370 diversity of xenobiotic compounds over the last few decades [41]. As a result,

371 current populations have evolved a myriad of genetic, behavioral and physiological

372 adaptive mechanisms, which could enhance their tolerance to new insecticides

$373[42,43]$. Our findings show that this scenario is happening with clothianidin whose

374 toxicity is reduced in some regions where agricultural activities are contributing to

375 the selection of resistant Anopheles populations.

377 WHO guidelines for clothianidin resistance testing in mosquitoes are yet to be 378 established and published. Assessing clothianidin susceptibility in Anopheles 379 mosquitoes using conventional bioassays has been met with some technical 380 challenges so far $[18,22,35,37,44]$. Clothianidin is difficult to solubilize in solvents 381 commonly used in bioassays, which has made the design of standard protocols more 382 laborious than usual [18,35,37]. In addition, because this active ingredient acts 383 slowly, resistance profiles are revealed via laboratory bioassays only if exposed 384 populations are rigorously monitored during a long holding period, which can last 385 seven days or more. Despite these difficulties, the susceptibility of wild Anopheles 386 populations from several African countries has been successfully evaluated using 387 the recommended diagnostic doses of clothianidin [18,22,35,37]. Here we have used 388 two protocols implemented and tested previously to analyze thousands of wild 389 mosquitoes and to provide the first evidence of clothianidin resistance in 
390 anopheline populations. In past studies, standard laboratory bioassays were used to 391 assess the susceptibility profile of the most important vectors of Plasmodium 392 parasites in Sub-Saharan Africa-An. gambiae, An. coluzzii, An. funestus and An.

393 arabiensis [18,22,35,37]. Resistance to clothianidin was not detected among 394 populations from 16 countries where $100 \%$ mortality was reached on average $72 \mathrm{~h}$

395 following exposure in all populations tested either with WHO tube tests [18,37] or 396 with CDC bottle assays [22,35]. Our study confirms that susceptible populations of 397 An. coluzzii and An. gambiae die on average within $72 \mathrm{~h}$ of holding period. Of note, 398 An. coluzzii samples, collected from the most urbanized areas of Yaoundé where 399 populations thrive in polluted environments and are highly resistant to several 400 classes of insecticides [28,31,45,46], remained susceptible to clothianidin. A recent 401 study carried out in Ivory Coast also showed that An. coluzzii populations from 402 agricultural sites were susceptible to clothianidin. These data suggest that this 403 species may have not yet developed resistance mechanisms against this active 404 ingredient [22].

406 By contrast to previous investigations, we observed resistance to clothianidin in $A n$. 407 gambiae with mortality rates as low as $46.5 \%$ among populations from an 408 agricultural region. Populations from surrounding locations remained largely 409 susceptible. Overall, our findings and other previous surveys suggest that 410 clothianidin resistance is present in anopheline populations, but is likely not yet 411 widespread among species and geographic areas [18]. Furthermore, the number of 412 registered neonicotinoids and probably their usage vary between African countries 413 and among geographic areas within countries [25-27]. Such variability may also 414 lead to geographic heterogeneity in selection pressure and could partly explain why 415 resistance is not detected in some settings. The development of clothianidin 416 resistance in An. gambiae in an agricultural region as shown here is not very 417 surprising albeit potentially harmful to malaria control efforts. Indeed, there is a 418 long list of observations providing indirect evidence that intensive and unrestricted 419 application of agricultural pesticides contributes to the selection of malaria vector 420 populations with reduced susceptibility to some active ingredients used in public 
421 health programs (reviewed in[23]). In Sub-Saharan Africa, resistance to pyrethroids,

422 organophosphates, carbamates and organochlorines is particularly strong among

423 populations from agricultural regions where a wide spectrum of pesticides is

424 routinely used for crop protection (e.g., $[19,20,47,48])$. During our field work, we

425 found evidence of usage of some formulations containing imidacloprid and

426 acetamiprid respectively, but there is a larger number of neonicotinoid formulations

427 available on local markets across Cameroon [25]. However, it is also important to

428 remind that Culex populations collected from standing water within farm plots in

429 Nkolondom were susceptible to clothianidin. This implies that exposure to

430 agricultural neonicotinoids does not automatically lead to resistance in mosquitoes

431 but could trigger other adaptive mechanisms that are yet to be elucidated. A direct

432 test of the association between agricultural neonicotinoids and the development of

433 resistance to clothianidin in Anopheles is beyond the scope of the present work and

434 should be the subject of future studies involving observational and experimental

435 approaches. In addition, one caveat of the present study is that sampling was limited

436 to only one agricultural region, and further investigation is needed to draw robust

437 conclusions about the current situation of clothianidin resistance in African

438 countries. Nonetheless, it is conceivable that in farms such as that of Nkolondom, the

439 application of neonicotinoids selects clothianidin-resistant populations, which

440 proliferate year-round in abundant breeding sites created by permanent irrigation.

442 Our results show that resistance to clothianidin is already strong in An. gambiae 443 populations from the Nkolondom farm where low mortality rates are observed even 444 with high doses of the active ingredient. Indeed, urban agriculture has been 445 intensified in the swampy area of this village since the 1980's during the economic 446 depression in Cameroon [29]. As a result, mosquito populations have likely had 447 enough time to develop such high levels of resistance. Bioassay tests using CDC 448 bottles coated with the synergist PBO in addition to clothianidin showed an increase 449 in mortality up to $90 \%$ in resistant populations. This suggests that Cytochrome P450 450 (CYPs) play a primarily role in clothianidin resistance in Anopheles gambiae. This 451 finding is in keeping with a large body of previous work, which revealed that 
452 clothianidin resistance in wild populations of many crop pests, primarily those of

453 the order Hemiptera (aphids, whiteflies, and planthoppers), is associated with

454 overexpression of one or several CYP enzymes [49-52]. Nevertheless, the strength

455 of resistance observed in Nkolondom suggests that the underlying mechanisms

456 might be more complex. Based on insights gained from studying the spread of

457 pyrethroid resistance in malaria vector populations across Africa, such a high level

458 of resistance to clothianidin could involve mutations at the target site and

459 modifications of the insect cuticle in addition to the prime role played by enzymatic

460 detoxification [53].

462 LLINs coated with pyrethroids have been widely distributed throughout Cameroon 463 over the last decade [54]. Although no IRS campaign has been carried out in the 464 country so far, the National Malaria Control Program and PMI have identified the 465 North and Far North, as the promising targets for a future implementation of IRS 466 [55]. Most anopheline populations across the country are already highly resistant to 467 pyrethroids and sometimes to several other classes of insecticide including 468 carbamates, organophosphates and organochlorines (reviewed in [56]). In such 469 multi-resistant populations, cross-resistance to insecticides with similar modes of 470 action may contribute to the build-up of tolerance to new insecticides [57]. As 471 clothianidin has a distinct mode of action, cross-resistance to the other insecticides 472 in use in malaria vector control so far is unlikely $[8,13]$. However, one source of 473 concern is that CYPs enzymes, which are already overexpressed in anopheline 474 populations throughout the continent due to widespread use of pyrethroids, could 475 enhance metabolic resistance against clothianidin. Nevertheless, our data also show 476 that populations from urban areas in which overexpression of CYPs and other 477 metabolic enzymes has been previously documented remain fully susceptible to 478 clothianidin $[31,45,58]$. Overall, although the underlying mechanisms rely heavily 479 on detoxification enzymes of the CYPs family that are ubiquitous among multi480 resistant vector populations, clothianidin resistance remains clustered around the 481 hotspot of agricultural usage of neonicotinoids. We thus argue that clothianidin 482 remains a potentially effective insecticide against pyrethroid-resistant malaria 
483 vector populations in Cameroon if it is not used in areas such as the Nkolondom

484 farm where the likelihood of operational failure increases. Such agricultural regions

485 should be mapped in IRS target areas prior to the rollout of clothianidin

486 formulations. Importantly, reliable bioassay protocols for clothianidin resistance

487 testing in Anopheles are now available [18,22,35] and could ease the scale up of

488 resistance monitoring by national malaria control programs. Mapping the sources of

489 selection pressure, which could contribute to the emergence and development of

490 resistance among vector populations in IRS target areas, is crucial to the successful

491 use of new IRS formulations.

\section{Conclusions}

495 This study shows that the novel insecticide clothianidin may have reduced efficacy 496 in some agricultural regions of Sub-Saharan Africa due to pre-existing levels of 497 resistance among target populations. The decision-making process regarding the 498 potential deployment of new active ingredients in malaria vector control programs 499 has been mainly based upon selecting compounds with a unique mode of action so 500 as to prevent cross-resistance between insecticides in current use in public health.

501 The present work suggests that before new insecticides are considered for inclusion

502 in National Malaria Control Program resistance management strategies, their

503 efficacy should be particularly monitored in areas where similar compounds are 504 being used in agriculture.

505 


\section{Figure legends}

508

509 Figure 1: Description of the study site. (A) Map of the neighborhoods of Yaoundé

510 where clothianidin susceptibility of Anopheles and Culex mosquitoes was evaluated.

511 (B) A typical vegetable plot of the Nkolondom farm. (C) Used cans of imidacloprid

512 and acetamiprid collected from the fields. (B) Application of pesticide mixtures on a

513 vegetable plot in Nkolondom

514
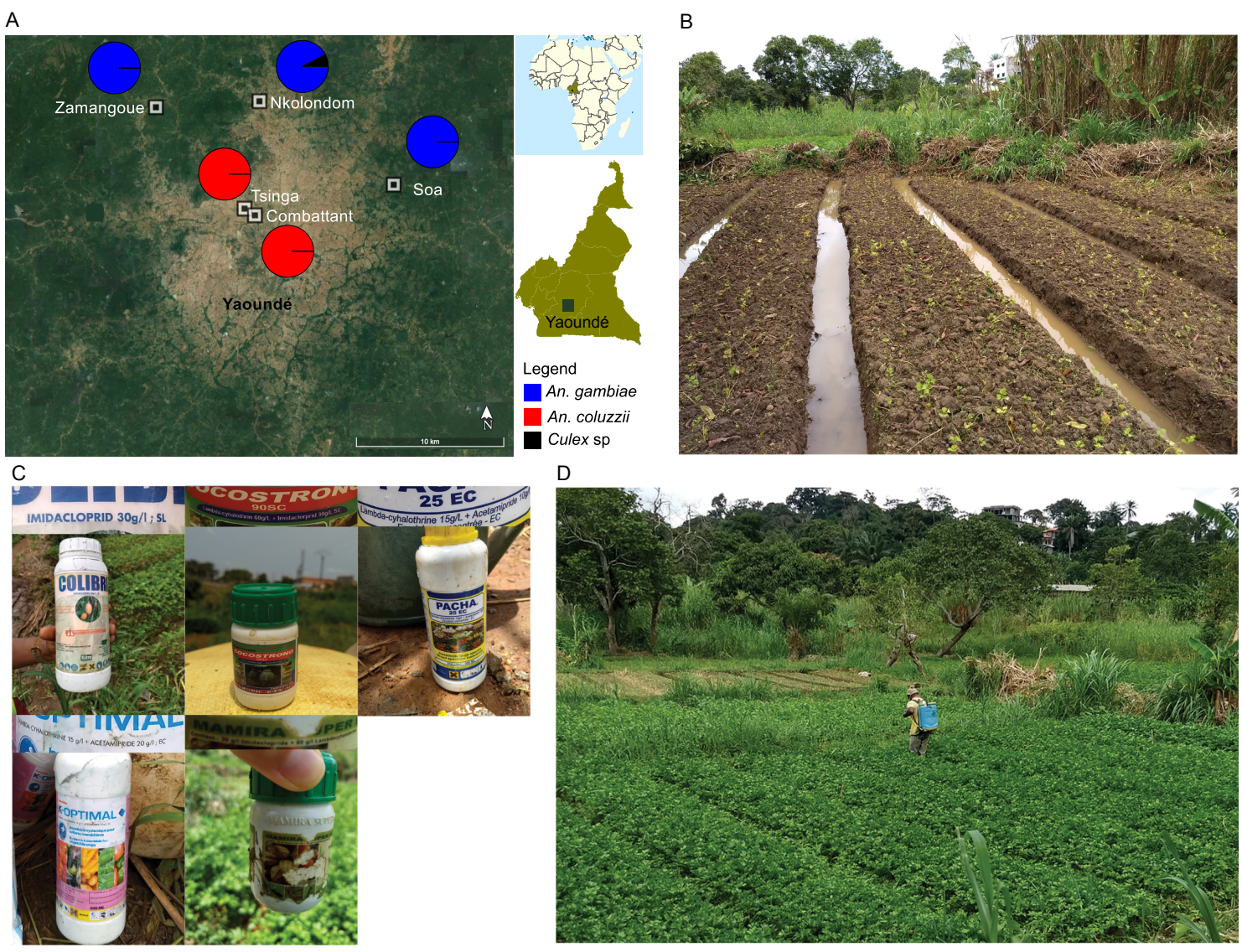
516 Figure 2: Susceptibility to clothianidin in laboratory strains and field populations.

517 (A) Mortality of Anopheles and Culex populations exposed to $150 \mu \mathrm{g} / \mathrm{ml}$ clothianidin

518 in CDC bottles. (B) A gradient of clothianidin susceptibility in wild populations of $A n$.

519 gambiae. (C) Susceptibility of a laboratory strain and of Nkolondom populations to

520 SumiShield 50WG evaluated with WHO tube tests. (D) Dramatic increase in

521 clothianidin toxicity in Nkolondom populations in the presence of the synergist PBO.

522

523

524
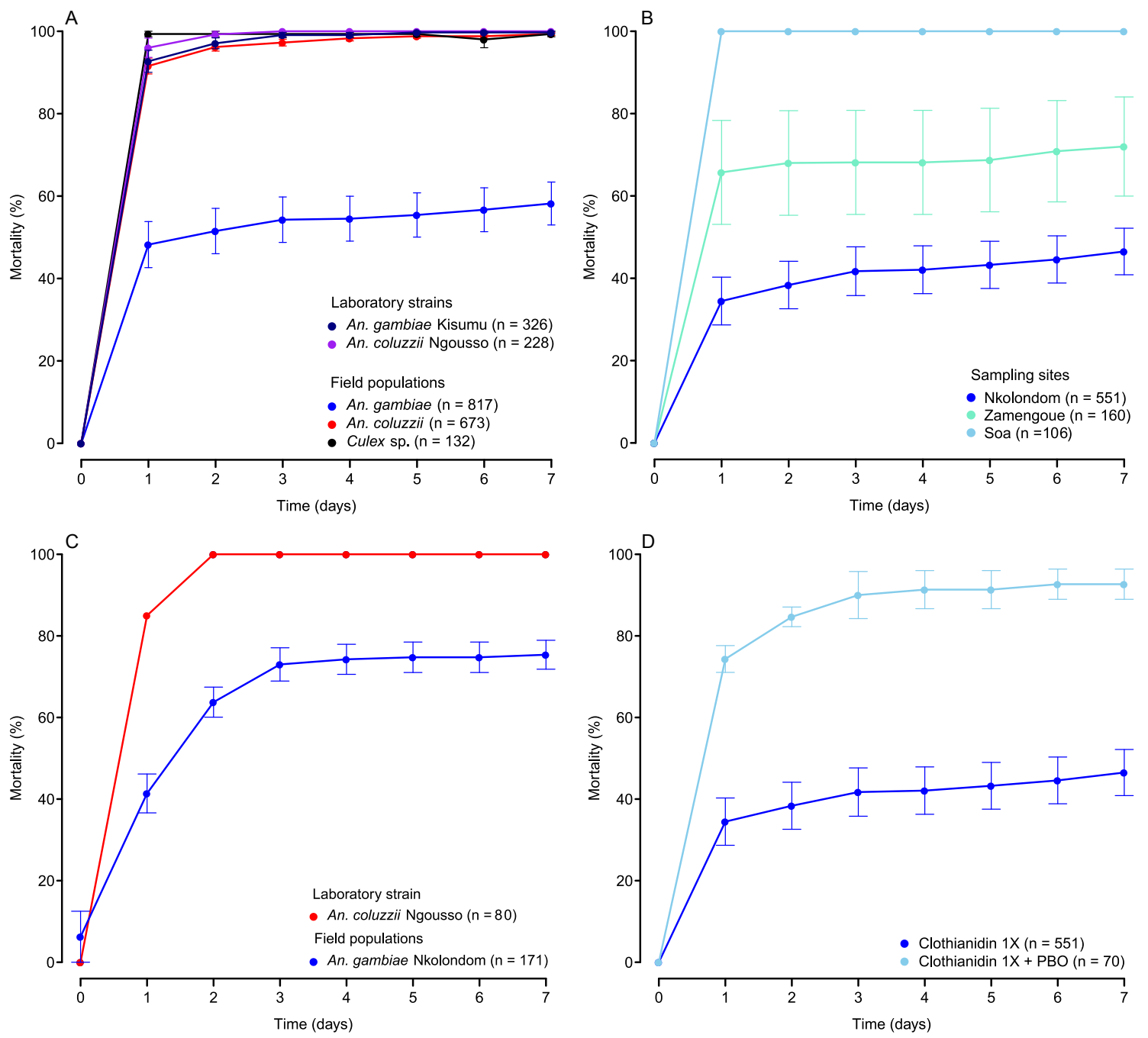

525 
526 Figure 3: Knockdowns after 60 min exposure and susceptibility to increasing doses

527 of clothianidin. (A) Knockdowns in Anopheles and Culex populations in CDC bottle

528 bioassays. (B) Knockdowns in An. gambiae field populations. (C) Mortality of

529 mosquitoes from the Nkolondom farm exposed to $1 \mathrm{X}, 5 \mathrm{X}$ and 10X the active dose of

530 clothianidin in CDC bottles. Standard errors are shown..
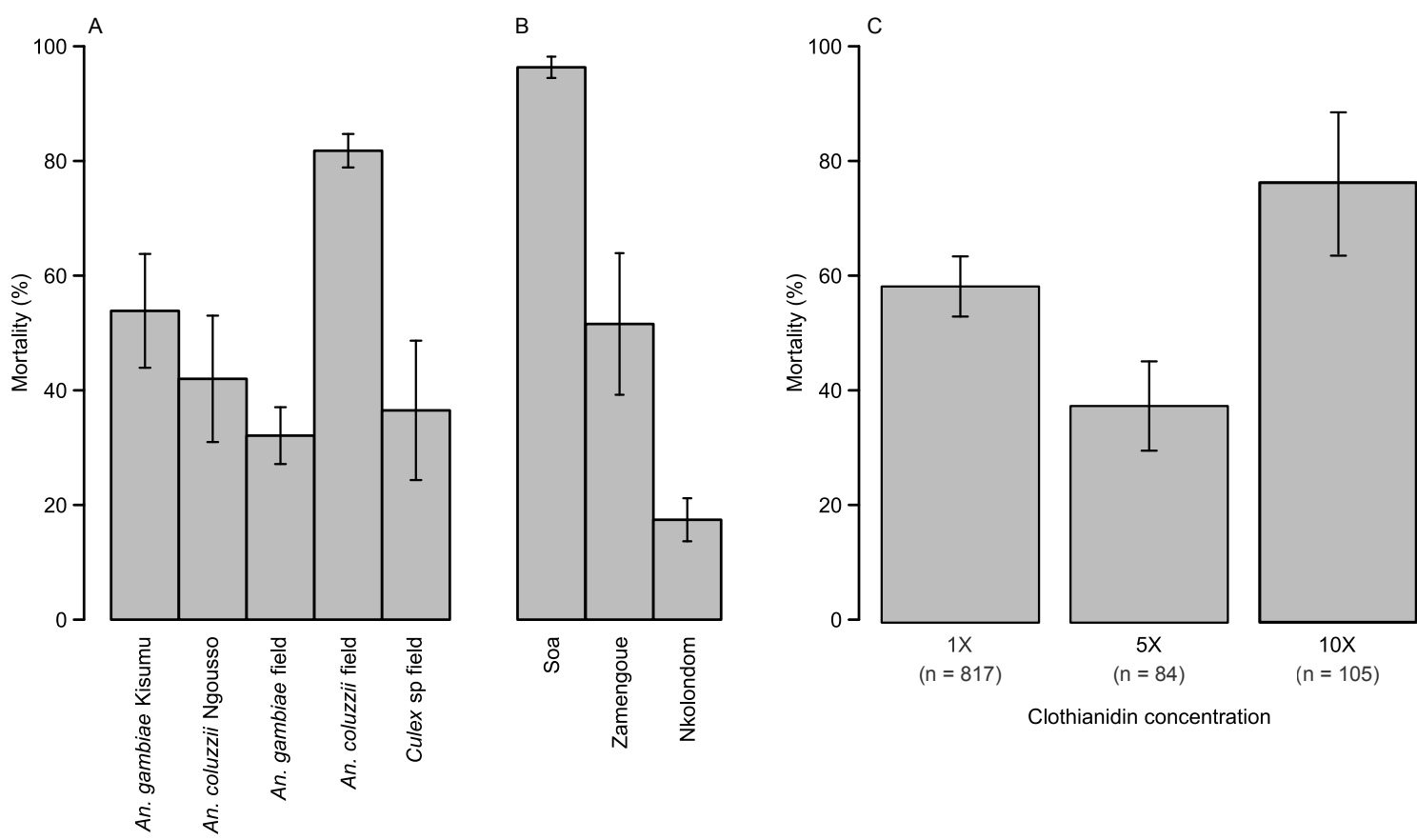

532 


\section{Authors' contributions}

546 CF, CK and CSW designed the study. CF, WT, MA, FA and CK carried out

547 entomological collections and laboratory tests. CF and CK analyzed the data and

548 drafted the manuscript, which was revised by co-authors. All authors read and

549 approved the final manuscript.

550

\section{Acknowledgements}

552 The authors would like to thank residents of Nkolondom and especially farmers for

553 their cooperation during fieldwork.

554

\section{Competing interests}

556 The authors declare that they have no competing interests.

\section{Availability of data and materials}

559 Not applicable.

560

\section{Consent for publication}

562 Not applicable.

563

\section{Ethics approval and consent to participate}

565 The study protocol was reviewed and approved by the Regional Ethics Committee

566 for Human health Research of Ministry of Public Health Cameroon (1-

567 141/CRERSHC/2020).

568

569 Funding

570 This study was supported by a Royal Society fellowship ((FLAIR) FLR \R1 \201216)

571 and National Institutes of Health grant (R01AI150529) to CK. The content is solely

572 the responsibility of the authors and does not necessarily represent the official

573 views of the National Institutes of Health. 


\section{References}

577 1. Hemingway J. The way forward for vector control. Science (80-. ). 2017;358:998-

5789.

579 2. Beier JC, Wilke ABB, Benelli G. Newer Approaches for Malaria Vector Control and

580 Challenges of Outdoor Transmission. Towar. Malar. Elimin. - A Leap Forw.

581 IntechOpen; 2018.

582 3. Bhatt S, Weiss DJ, Cameron E, Bisanzio D, Mappin B, Dalrymple U, et al. The effect

583 of malaria control on Plasmodium falciparum in Africa between 2000 and 2015.

584 Nature. 2015;526:207-11.

585 4. Hemingway J, Ranson H, Magill A, Kolaczinski J, Fornadel C, Gimnig J, et al.

586 Averting a malaria disaster: Will insecticide resistance derail malaria control?

587 Lancet. 2016;387:1785-8.

588 5. Kleinschmidt I, Bradley J, Knox TB, Mnzava AP, Kafy HT, Mbogo C, et al.

589 Implications of insecticide resistance for malaria vector control with long-lasting

590 insecticidal nets : a WHO-coordinated, prospective, international, observational

591 cohort study. Lancet Infect. Dis. 2018;18:640-9.

592 6. World Health Organization. Global Plan for Insecticide Resistance Management in

593 Malaria Vectors. Geneva: World Health Organization; 2012.

594 7. Mnzava AP, Knox TB, Temu EA, Trett A, Fornadel C, Hemingway J, et al.

595 Implementation of the global plan for insecticide resistance management in malaria

596 vectors: Progress, challenges and the way forward. Malar. J. 2015;14:1-9.

597 8. WHO. List of WHO Prequalified Vector Control Products. 2020.

598 9. WHO. Guidelines for malaria vector control. 2019.

599 10. WHO. Malaria Fact Sheets. 2020;

600 11. Bayer. Fludora fusion [Internet]. [Internet]. [cited 2020 Apr 9]. Available from:

601 https://www.vectorcontrol.bayer.com/solutions/products/fludora-fusion

602 12. Sumitomo Chemical. SumiShield 50WG [Internet]. [cited 2020 Apr 9]. Available

603 from: https://www.sumivector.com/sumishield-50wg

604 13. Simon-delso N, Amaral-rogers V, Belzunces L, Bonmatin JM, Chagnon M, Downs

605 C, et al. Systemic insecticides (neonicotinoids and fipronil): trends, uses, mode of 606 action and metabolites. Environ. Sci. Pollut. Res. 2015;22:5-34. 
607 14. Agossa FR, Padonou GG, Fassinou AJYH, Odjo EM, Akuoko OK, Salako A, et al.

608 Small-scale field evaluation of the efficacy and residual effect of Fludora ${ }^{\circledR}$ Fusion

609 (mixture of clothianidin and deltamethrin) against susceptible and resistant

610 Anopheles gambiae populations from Benin, West Africa. Malar. J. BioMed Central;

$6112018 ; 17: 484$.

612 15. Agossa FR, Padonou GG, Koukpo CZ, Zola-Sahossi J, Azondekon R, Akuoko OK, et

613 al. Efficacy of a novel mode of action of an indoor residual spraying product,

614 SumiShield $® 50 W G$ against susceptible and resistant populations of Anopheles

615 gambiae (s.l.) in Benin, West Africa. Parasites and Vectors. Parasites \& Vectors;

616 2018;11:1-13.

617 16. Kweka E, Mahande A, Ouma J, Msangi S, Karanja W, Temba V, et al. Novel Indoor

618 Residual Spray Insecticide With Extended Mortality Effect : A Case of SumiShield

619 50WG Against Wild Resistant Populations of Anopheles arabiensis in Northern

620 Tanzania. Glob. Heal. Sci. Pract. 2018;6:758-65.

621 17. Uragayala S, Kamaraju R, Tiwari SN, Sreedharan S, Ghosh SK, Valecha N. Village-

622 scale ( Phase III) evaluation of the efficacy and residual activity of SumiShield 50®

623 WG ( Clothianidin 50 \%, w/w ) for indoor spraying for the control of pyrethroid-

624 resistant Anopheles culicifacies Giles in Karnataka state , India. Trop. Med. Int. Heal.

$6252018 ; 23: 605-15$.

626 18. Oxborough RM, Seyoum A, Yihdego Y, Dabire R, Gnanguenon V, Wat F, et al.

627 Susceptibility testing of Anopheles malaria vectors with the neonicotinoid

628 insecticide clothianidin; results from 16 African countries, in preparation for indoor

629 residual spraying with new insecticide formulations. Malar. J. BioMed Central;

630 2019;18:https://doi.org/10.1186/s12936-019-2888-6.

631 19. Hien AS, Soma DD, Hema O, Bayili B, Namountougou M, Gnankine O, et al.

632 Evidence that agricultural use of pesticides selects pyrethroid resistance within

633 Anopheles gambiae s. 1. populations from cotton growing areas in Burkina Faso ,

634 West Africa. PLoS One. 2017;12:1-15.

635 20. Nkya TE, Akhouayri I, Poupardin R, Batengana B, Mosha F, Magesa S, et al.

636 Insecticide resistance mechanisms associated with different environments in the

637 malaria vector Anopheles gambiae: a case study in Tanzania. Malar. J. 2014;13:28. 
638 21. Diabate A, Baldet T, Chandre F, Akogbeto M, Guiguemde RT, Darriet F, et al. The

639 Role Of Agricultural Use Of Insecticides In Resistance To Pyrethroids In Anopheles

640 gambiae s .1 . In Burkina Faso. Am. J. Trop. Med. Hyg. 2002;67:617-22.

641 22. Mouhamadou CS, Souza SS De, Fodjo BK, Zoh MG, Bli NK, Koudou BG. Evidence of

642 insecticide resistance selection in wild Anopheles coluzzii mosquitoes due to

643 agricultural pesticide use. Infect. Dis. Poverty. Infectious Diseases of Poverty;

$644 \quad 2019 ; 8: 1-8$.

645 23. Reid MC, McKenzie FE. The contribution of agricultural insecticide use to

646 increasing insecticide resistance in African malaria vectors. Malar. J. BioMed Central;

$647 \quad 2016 ; 15: 107$.

648 24. Bradford BZ, Huseth AS, Groves RL. Widespread detections of neonicotinoid

649 contaminants in central Wisconsin groundwater. PLoS One. 2018;13:e0201753.

650 25. Ngamo Tinkeu L. Current situation on the registration and use of neonicotinoids

651 in Cameroon. https ://www.interacademies.org/49035/Cameroon-by-Leonard-

652 Ngamo-Tinke u-University-of-Ngaoundr. 2018.

653 26. Katambo M. Neonicotinoids and their impact on ecosystem services in Tanzania.

654 http://www.interacademies.org/49301/Neonicotinoids-in-Tanzania-by-Mkabwa-

655 Katambo. 2018.

656 27. Akpesse A. Neonicotinoids and their impact on ecosystem service for agriculture 657 and biodiversity in Africa. https ://www.interacademies.

658 org/49037/Neonicotinoids-at-Cote-dIvoire-by-Akpa-Akpesse-University-Flix-

659 HouphoutBoigny. 2018.

660 28. Kamdem C, Tene Fossog B, Simard F, Etouna J, Ndo C, Kengne P, et al.

661 Anthropogenic Habitat Disturbance and Ecological Divergence between Incipient

662 Species of the Malaria Mosquito Anopheles gambiae. PLoS One. 2012. p. e39453.

663 29. Temple L, Marquis S, David O, Simon S. Le maraîchage périurbain à Yaoundé est-

664 il un système de production localisé innovant? Econ. Sociétés. Série AG Systèmes

665 Agroaliment. Press. l'ISMEA. 2008;30:2309-28.

666 30. Service MW. Mosquito ecology: field sampling methods. 2nd ed. Netherlands:

667 Springer; 1993.

668 31. Kamdem C, Fouet C, Gamez S, White BJ. Pollutants and insecticides drive local 
669 adaptation in African malaria mosquitoes. Mol. Biol. Evol. 2017;34:1261-75.

670 32. Gillies MT, Coetzee M. A supplement to the Anophelinae of Africa south of the

671 Sahara. Johannesburg: The South African Institute for Medical Research; 1987.

672 33. Fanello C, Santolamazza F, DellaTorre A. Simultaneous identification of species

673 and molecular forms of the Anopheles gambiae complex by PCR-RFLP. Med. Vet.

674 Entomol. 2002;16:461-4.

675 34. Cdc. Guideline for Evaluating Insecticide Resistance in Vectors Using the CDC

676 Bottle Bioassay. CDC Methods. 2012;1-28.

677 35. Agumba S, Gimnig JE, Ogonda L, Ombok M, Kosgei J, Munga S, et al. Diagnostic

678 dose determination and efficacy of chlorfenapyr and clothianidin insecticides

679 against Anopheles malaria vector populations of western Kenya. Malar. J. BioMed

680 Central; 2019;18:1-9.

681 36. World Health Organization. Test procedures for insecticide resistance

682 monitoring in malaria vector mosquitoes. World Health Organization, editor. World

683 Heal. Organ. Tech. Rep. Ser. World Health Organization; 2016.

684 37. Dagg K, Irish S, Wiegand RE, Shililu J, Yewhalaw D, Messenger LA. Evaluation of

685 toxicity of clothianidin (neonicotinoid) and chlorfenapyr (pyrrole) insecticides

686 and cross-resistance to other public health insecticides in Anopheles arabiensis

687 from Ethiopia. Malar. J. BioMed Central; 2019;18:1-11.

688 38. Video protocol showing how to treat filter papers with clothianidin.

689 39. Bernard C, Hilogène B. Insecticide synergists: role, importance, and perspectives.

$690 \quad J$ Toxicol Env. Heal. 1993;38:199-223.

691 40. R Core Team. R: A language and environment for statistical computing. Vienna,

692 Austria: R Foundation for Statistical Computing; 2016.

693 41. Fouet C, Atkinson P, Kamdem C. Human Interventions: Driving Forces of

694 Mosquito Evolution. Trends Parasitol. Elsevier Ltd; 2018;34:127-39.

695 42. Miles A, Harding NJ, Bottà G, Clarkson CS, Antão T, Kozak K, et al. Genetic

696 diversity of the African malaria vector anopheles gambiae. Nature. 2017;552:96-

697100.

698 43. Gatton ML, Chitnis N, Churcher T, Donnelly MJ, Ghani AC, Charles HJ, et al. The

699 importance of moquito behavioural adaptations to malaria control in Africa. 
$700 \quad$ Evolution. 2013;67:1218-30.

701 44. Darriet F, Chandre F. Efficacy of six neonicotinoid insecticides alone and in

702 combination with deltamethrin and piperonyl butoxide against pyrethroid-resistant

703 Aedes aegypti and Anopheles gambiae ( Diptera : Culicidae ). Pest Manag. Sci.

704 2013;69:905-10.

705 45. Fossog Tene B, Poupardin R, Costantini C, Awono-Ambene P, Wondji CS, Ranson

$706 \mathrm{H}$, et al. Resistance to DDT in an Urban Setting: Common Mechanisms Implicated in

707 Both M and S Forms of Anopheles gambiae in the City of Yaoundé Cameroon. PLoS

708 One. $2013 ; 8$.

709 46. Antonio-Nkondjio C, Fossog BT, Ndo C, Djantio BM, Togouet SZ, Awono-Ambene

$710 \mathrm{P}$, et al. Anopheles gambiae distribution and insecticide resistance in the cities of

711 Douala and Yaoundé (Cameroon): influence of urban agriculture and pollution.

712 Malar. J. BioMed Central Ltd; 2011;10:154.

713 47. Djogbénou LS, Assogba B, Djossou L, Makoutode M. Indirect evidence that

714 agricultural pesticides select for insecticide resistance in the malaria vector

715 Anopheles gambiae. J. Vector Ecol. 2016;41:34-40.

716 48. Nwane P, Etang J, Chouaibou M, Toto JC, Kerah-hinzoumbé C, Mimpfoundi R, et

717 al. Trends in DDT and pyrethroid resistance in Anopheles gambiae s.s. populations

718 from urban and agro-industrial settings in southern Cameroon. BMC Infect. Dis.

719 2009;9:doi:10.1186/1471-2334-9-163.

720 49. Jin R, Mao K, Liao X, Xu P, Li Z, Ali E, et al. Overexpression of CYP6ER1 associated

721 with clothianidin resistance in Nilaparvata lugens (Stål). Pestic. Biochem. Physiol.

722 Elsevier; 2019;154:39-45.

723 50. Bass C, Puinean AM, Andrews M, Cutler P, Daniels M, Elias J, et al. Mutation of a

724 nicotinic acetylcholine receptor $\beta$ subunit is associated with resistance to

725 neonicotinoid insecticides in the aphid Myzus persicae. BMC Neurosci. 2011;12.

726 51. Yang N, Xie W, Jones CM, Bass C, Jiao X, Yang X, et al. Transcriptome profiling of

727 the whitefly Bemisia tabaci reveals stage-specific gene expression signatures for

728 thiamethoxam resistance. Insect Mol. Biol. 2013;22:485-496.

729 52. Abdallah IS, Abou-yousef HM, Fouad EA, Kandil MAE. The role of detoxifying

730 enzymes in the resistance of the cowpea aphid (Aphis craccivora Koch ) to 
731 thiamethoxam. J. Plant Prot. Res. 2016;56:67-72.

732 53. Ranson H, Lissenden N. Insecticide Resistance in African Anopheles Mosquitoes:

733 A Worsening Situation that Needs Urgent Action to Maintain Malaria Control.

734 Trends Parasitol. Elsevier Ltd; 2016;32:187-96.

735 54. Bowen HL. Impact of a mass media campaign on bed net use in Cameroon. Malar.

736 J. Malaria Journal; 2013;12:36.

737 55. President's Malaria Initiative Cameroon. Malaria Operational Plan FY 2017.

7382017.

739 56. Antonio-nkondjio C, Sonhafouo-Chiana N, Ngadjeu CS, Doumbe-Belisse P,

740 Talipouo A, Djamouko-Djonkam L, et al. Review of the evolution of insecticide

741 resistance in main malaria vectors in Cameroon from 1990 to 2017. Parasites and

742 Vectors. Parasites \& Vectors; 2017;10:1-14.

743 57. Hemingway J, Ranson H. Insecticide Resistance In Insect Vectors Of Human

744 Disease. Annu. Rev. Entomol. 2000;45:371-91.

745 58. Cassone BJ, Kamdem C, Cheng C, Tan JC, Hahn MW, Costantini C, et al. Gene

746 expression divergence between malaria vector sibling species Anopheles gambiae

747 and An. coluzzii from rural and urban Yaoundé Cameroon. Mol. Ecol. 2014;23:2242- 